\title{
In Situ Ultrahigh Vacuum Transmission Electron Microscope Investigations of Nanostructures
}

L. J. Chen* and W.W. Wu**

*Department of Materials Science and Engineering, National Tsing Hua University, Hsinchu 300, Taiwan, Republic of China

**Department of Materials Science and Engineering, National Chiao Tung University, Hsinchu 300, Taiwan, Republic of China

Low-dimensional nanostructures, such as nanodots, nanowires and nanotubes, are especially attractive candidates for developing a bottom-up paradigm for nanotechnology-enabled architectures. In situ ultrahigh vacuum transmission electron microscope (UHV-TEM) is a powerful tool to investigate the dynamic changes of nanostructures. By observing growth and phase transitions in situ, understanding of their mechanisms can be used to model relevant processes. Recent development of UHV-TEM further enables the investigation on atomic-scale materials systems in a clean environment. With the precise knowledge of the changes occurred on an atomic level, accurate control of the growth process can be achieved [1]. The appropriate utilization of the UHV-TEM will be beneficial in studying the fundamental mechanisms of dynamic reactions, formation of transient phase, solid-state amorphization, epitaxial growth, growth kinetics and evolution of defects.

The dynamical changes occurred on the nano scale are often unexpected, which also underscores the importance of the approach. In this presentation, we will highlight several examples to demonstrate the unique capability of in situ TEM to study the dynamical changes. The examples include growth of high-density titanium silicide nanowires in a single direction on a silicon surface [2], controlled growth of atomic-scale Si layer with huge strain in the nano-heterostructure $\mathrm{NiSi} / \mathrm{Si} / \mathrm{NiSi}$ through point contact reaction between nano-wires of $\mathrm{Si}$ and $\mathrm{Ni}$ and reactive epitaxial growth [3], observation of repeating events of nucleation in epitaxial growth of nano $\mathrm{CoSi}_{2}$ in nanowires of $\mathrm{Si}$ [4] and observations of electromigration in copper lines at room temperature [5].

\section{References}

[1] F. M. Ross, R. M. Tromp, and M. C. Reuter, Science 286 (1999) 1931.

[2] H. C. Hsu, W. W. Wu, H. F. Hsu, and L. J. Chen, Nano Lett. 7 (2007) 885.

[3] K. C. Lu, W. W. Wu, H. W. Wu, C. M. Tanner, J. P. Chang, L. J. Chen, and K. N. Tu, Nano Letts. 7 (2007) 2389.

[4] Y.C. Chou, W.W. Wu, S.L. Cheng, B.Y. Yoo, N. Myung, L.J. Chen, and K. N. Tu, Nano Lett. 8 (2008) 2194.

[5] K.C. Chen, W.W. Wu, C.N. Liao, L.J. Chen, and K.N. Tu, Science 321 (2008) 1066.

[6] The research was supported by the National Science Council through grants No. NSC 97-2120-M-007-003. 

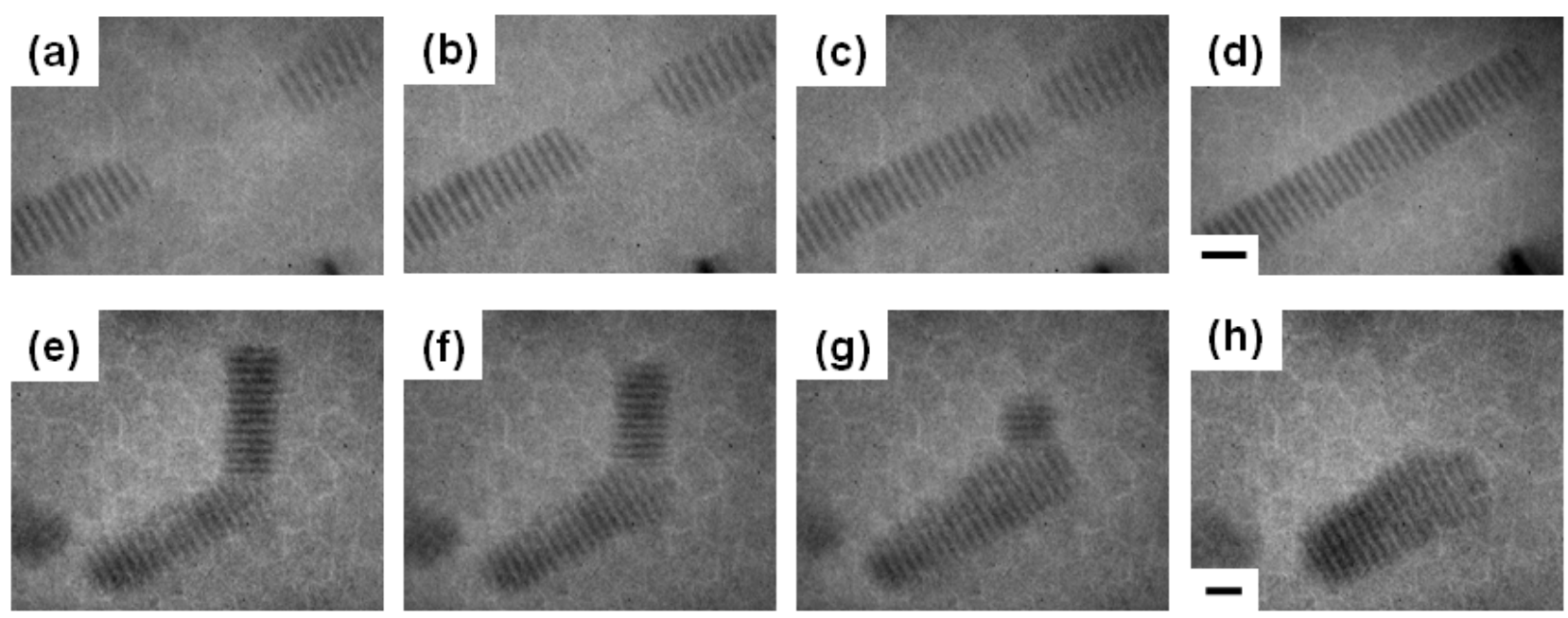

Fig. 1. In situ TEM images of the interactions of titanium silicide NWs at $700{ }^{\circ} \mathrm{C}$. The images of NWs in the same direction were obtained at elapsed times of (a) $0 \mathrm{~min}$, (b) $9 \mathrm{~min}$, (c) $18 \mathrm{~min}$, and (d) 19 min. The images of NWs in different directions were obtained at elapsed times of (e) $0 \mathrm{sec}$, (f) $25 \mathrm{sec}$, (g) $49 \mathrm{sec}$, and (h) $80 \mathrm{sec}$. The scale bar corresponds to $6 \mathrm{~nm}$ in (d) and $4 \mathrm{~nm}$ in (h).
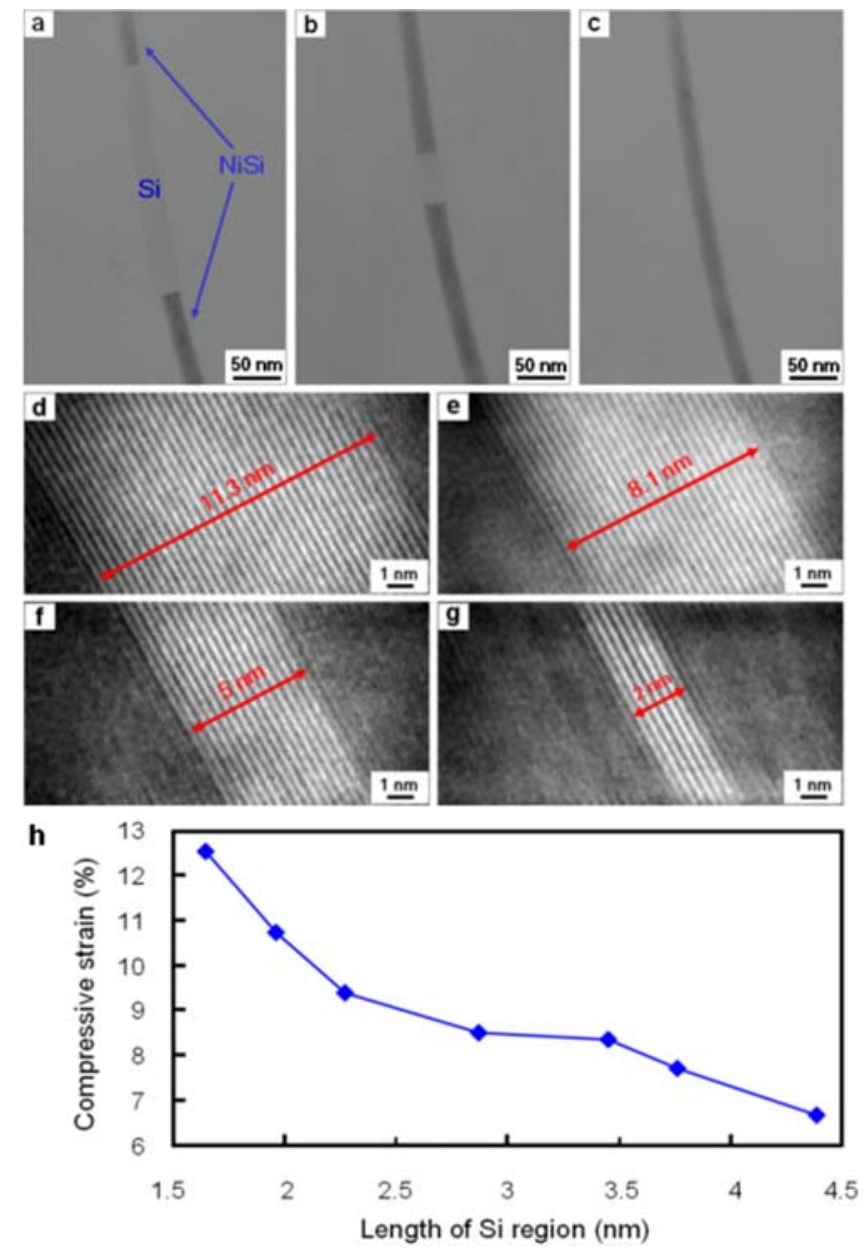

Fig. 2. In-situ TEM images showing the formation of NiSi/Si/NiSi heterostructures within a $\mathrm{Si}$ nanowire, and compressive strain in the Si region. a and b, In-situ TEM images of the NiSi/Si/NiSi heterostructure. The bright area is Si and the dark area is NiSi. d to g, High-resolution TEM images of $\mathrm{NiSi} / \mathrm{Si} / \mathrm{NiSi}$ heterostructures. The bright and dark portions of the lattice images correspond to $\mathrm{Si}$ and $\mathrm{NiSi}$, respectively. h, Plot of the compressive strain versus the length of the Si region in the nano-heterostructured $\mathrm{NiSi} / \mathrm{Si} / \mathrm{NiSi}$. 